\title{
Cucurbitacin E inhibits cellular proliferation and induces apoptosis in melanoma by suppressing HSDL2 expression
}

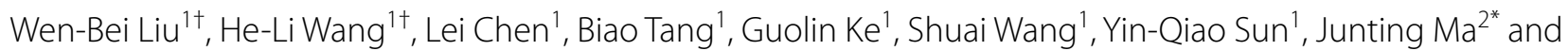
Da-Lun Lyu ${ }^{1 *}$

\begin{abstract}
Background: Melanoma is among the most aggressive types of skin malignancy and can have an unpredictable clinical course. Exploration of novel therapeutic targets and their regulators remains essential for the prevention and treatment of melanoma.

Methods: HSDL2 protein levels were examined by immunohistochemistry. The roles of HSDL2 in cell proliferation and apoptosis were identified by CCK-8 and colony formation assays. The function of HSDL2 in cell apoptosis was analysed by flow cytometry. Western blotting, cell proliferation and apoptosis and a xenograft tumour model were utilized to explore the inhibitory functions and mechanisms of CuE in melanoma.
\end{abstract}

Results: HSDL2 is overexpressed in melanoma and promotes melanoma progression by activating the ERK and AKT pathways. CUE could inhibit the ERK and AKT pathways by decreasing HSDL2 expression; therefore, CuE could inhibit melanoma growth in vitro and in vivo.

Conclusion: HSDL2 may be a promising therapeutic target against melanoma, and CuE can inhibit melanoma by downregulating HSDL2 expression.

Keywords: Melanoma, HSDL2, Cucurbitacin E, ERK and AKT pathways, Proliferation and apoptosis

\section{Introduction}

Skin cancer is the most common type of cancer in dermatology [1], and melanoma is one of the most fatal forms and is the fifth most common cancer in men and the sixth in women $[2,3]$. The incidence of melanoma has increased rapidly over past decades [4]. Although advances in surgical resection, radiation, and chemotherapy have improved the survival of cancer patients

\footnotetext{
*Correspondence: majunting@ahmu.edu.cn; Ivdalun0776@163.com tWen-Bei Liu and He-Li Wang ontributed equally to this study and are co-first authors

${ }^{1}$ Department of Dermato-Venerology and Department of Burn and Plastic Surgery, The First Affiliated Hospital of Wannan Medical College, Jinghu District, Wuhu 241000, Anhui, China

${ }^{2}$ Department of Pharmacology, School of Basic Medical Sciences, Anhui Medical University, Hefei 230032, Anhui, China
}

in recent decades, mortality rates remain high due to tumour recurrence and metastasis [5, 6]. Thus, the exploration of new effective molecular targets on tumour cells and their regulators would be useful for melanoma therapy.

Hydroxysteroid dehydrogenase-like (HSDL) 2 is a member of the short-chain dehydrogenase/reductase (SDR) superfamily that can catalyse the oxidation and reduction of various substrates, such as steroids, sugars, retinoids and fatty acids [7]. Previous studies have shown that HSDL2 is involved in lipid metabolism and the synthesis of cholesterol $[8,9]$, displaying significant protumour function by promoting cell proliferation and inhibiting apoptosis $[10,11]$, and has been reported to be associated with some cancers, such as pancreatic original author(s) and the source, provide a link to the Creative Commons licence, and indicate if changes were made. The images or other third party material in this article are included in the article's Creative Commons licence, unless indicated otherwise in a credit line to the material. If material is not included in the article's Creative Commons licence and your intended use is not permitted by statutory regulation or exceeds the permitted use, you will need to obtain permission directly from the copyright holder. To view a copy of this licence, visit http://creativecommons.org/licenses/by/4.0/. The Creative Commons Public Domain Dedication waiver (http://creativeco mmons.org/publicdomain/zero/1.0/) applies to the data made available in this article, unless otherwise stated in a credit line to the data. 
cancer, breast cancer, and papillary thyroid carcinoma [12-14]. However, to date, the function of HSDL2 in melanoma remains unclear.

Natural compounds found in vegetables, fruits, and medicinal plants have been considered potential sources of inhibitors for cancer [15]. Cucurbitacin E $(\mathrm{CuE})$ is a member of the cucurbitacin family, which is a group of tetracyclic triterpenoids extracted from cucurbitaceous plants [16]. Accumulated evidence has demonstrated that $\mathrm{CuE}$ has effective pharmacological properties, such as lipid reduction, hepatic protection, anti-inflammation, and antitumour activity [17]. The potential anticarcinogenic properties of $\mathrm{CuE}$ on diverse tumour types, such as gastric cancer, lung cancer, breast cancer and ovarian cancer, have been well studied [18-21]. However, the potential antitumour function and the underlying mechanisms of $\mathrm{CuE}$ in melanoma have not been fully elucidated.

In this paper, we found that HSDL2 promoted cell proliferation and suppressed apoptotic cell death by activating the AKT and ERK signalling pathways and that $\mathrm{CuE}$ could slow melanoma growth by inhibiting the AKT and ERK signalling pathways via the downregulation of HSDL2 expression.

\section{Material and methods}

\section{Reagents and chemicals}

HSDL2 (Cat: 15,631-1-AP, 1:1000) and $\alpha$-tubulin (Cat: 11,224-1-AP, 1:1000) were purchased from Proteintech (Wuhan, China). Bcl-2 (Cat: R22494; 1:1000), cleaved caspase-3 (Cat: 383,726; 1:1000), phospho-ERK (Cat: 301,245; 1:1000) and ERK (Cat: 201,245-4A4, 1:1000) were purchased from ZEN-BIOSCIENCE (Chengdu, China). AKT (Cat: A5031, 1:1000) was obtained from Bimake (Houston, TX, USA). Phospho-AKT (Cat: $4060 \mathrm{~T}, 1: 1000)$ was purchased from Cell Signaling Technology (Danvers, MA, USA). Cucurbitacin E was obtained from MedChemExpress (Cat: HY-N0417, New Jersey, USA).

\section{Clinical specimens}

Melanoma and adjacent normal tissue samples were obtained from 60 melanoma patients recruited from the First Affiliated Hospital of Wannan Medical College. The melanoma patients were between 31 and 76 years of age (mean, $50.3 \pm 18.4$ years) at diagnosis. Written informed consent was obtained from each patient, and the experimental protocol was approved by the Ethics Committee of the First Affiliated Hospital of Wannan Medical College. The clinicopathological characteristics of the samples are summarized in Table 1.
Table 1 Summary of clinicopathological characteristics

\begin{tabular}{lll}
\hline Characteristics & Category & Frequency \\
\hline Gender & Men/Women & $28 / 32$ \\
Age group & $\leq 50 />50$ & $26 / 34$ \\
Clinical stage & I+ II/ III IV & $40 / 20$ \\
Tumor thickness & $\leq 1 />1$ & $37 / 23$ \\
\hline
\end{tabular}

\section{Cell culture and lentivirus infection}

A375 human melanoma cell lines were purchased from the Cell Bank of the Chinese Academy of Sciences (Shanghai, China) and cultured in Dulbecco's modified Eagle's medium containing 10\% foetal bovine serum and $100 \mathrm{U} / \mathrm{ml}$ penicillin/streptomycin (all from Sangon Biotech, Shanghai, China) at $37^{\circ} \mathrm{C}$ in a $5 \% \mathrm{CO}_{2}$ incubator.

HSDL2-overexpressing lentivirus and shRNA targeting HSDL2 lentivirus was obtained from General Biol (Anhui, China). The sequences were as follows: human HSDL2, shRNA: 5'-CCA GAA GCA GTT AGC AAG AAA-3'; scrambled shRNA, shCtrl: $5^{\prime}$-TTC TCC GAA CGT GTC ACG T- $3^{\prime}$. Briefly, cells were seeded in 6-well plates and cultured at $37{ }^{\circ} \mathrm{C}$ and $5 \% \mathrm{CO} 2$ for $24 \mathrm{~h}$. The lentiviral particle suspension was added to the plate (multiplicity of infection $=5,5 \mu \mathrm{l}$ lentivirus per well). Following infection at $37^{\circ} \mathrm{C}$ for $12 \mathrm{~h}$, the culture medium was replaced. The cells were cultured for $72 \mathrm{~h}$, and infection efficiency was determined by real-time reverse transcription quantitative polymerase chain reaction (RT-qPCR) and western blotting assays.

\section{RT-qPCR}

Total RNA was extracted with TRIzol reagent (Beyotime, R0016) according to the manufacturer's instructions. Reverse transcription was performed using Moloney murine leukaemia virus reverse transcriptase (Promega, Madison, Wisconsin, USA) according to the manufacturer's protocol along with oligo(dT) primers (Sangon Biotech) to obtain cDNA. HSDL2 mRNA expression was examined with qPCR. The reaction consisted of $1 \times$ SYBR Green Master Mix (Sangon Biotech) and $0.06 \mu \mathrm{M}$ each primer under the following conditions: $94{ }^{\circ} \mathrm{C}$ for $5 \mathrm{~min}$, followed by 40 cycles of $94{ }^{\circ} \mathrm{C}$ for $15 \mathrm{~s}$ and $58{ }^{\circ} \mathrm{C}$ for $40 \mathrm{~s}$. Each sample was run in triplicate. The forward and reverse primers were synthesized by Sangon Biotech and had the following sequences: HSDL2, 5'-AAG CCA CTC AAG CAA TCT ATC TG-3' and $5^{\prime}$-GCT CTC CAT ATC CGA CAT TCC C-3'; and glyceraldehyde 3-phosphate dehydrogenase (GAPDH), $5^{\prime}$-TGA CTT CAA CAG CGA CAC CCA-3' and 5'-CAC CCT GTT GCT GTA GCC AAA-3'. The relative HSDL2 expression level was normalized to that of GAPDH and quantitated with the $2^{-\Delta \Delta C T}$ method. 


\section{Western blot analysis}

Cells were washed with phosphate-buffered saline (PBS) and then lysed in lysis buffer composed of $100 \mathrm{mM}$ Tris$\mathrm{HCl}$ (pH 6.8), $0.15 \mathrm{M} \mathrm{NaCl}, 5 \mathrm{mM}$ EDTA (pH 8.0), $1 \%$ Triton X-100, $5 \mathrm{mM}$ dithiothreitol, and $0.1 \mathrm{mM}$ phenylmethylsulfonyl fluoride for total protein extraction. Protein concentration was determined with the BCA Protein Assay Kit (Sangon Biotech); $20 \mu \mathrm{g}$ of the cell solution was mixed with $5 \times$ loading buffer and separated by sodium dodecyl sulfate-polyacrylamide gel electrophoresis on a $12.5 \%$ acrylamide gel. The proteins were transferred to a polyvinylidene difluoride membrane (Amersham Biosciences, Little Chalfont, UK) that was blocked with $5 \%$ skim milk dissolved in Tris-buffered saline with $0.1 \%$ Tween-20 (TBST) for $1 \mathrm{~h}$ at room temperature and then incubated with primary antibodies overnight at $4{ }^{\circ} \mathrm{C}$. After washing three times with TBST, the membrane was incubated with horseradish peroxidase (HRP)-conjugated secondary antibody (Santa Cruz Biotechnology), and immunoreactivity was detected using an ECL Western Blotting Substrate kit (Pierce, Rockford, IL, USA).

\section{Colony formation assay}

A375 cells at a density of 1000 cells/well were resuspended and seeded into six-well plates. After culturing for two weeks, the colonies were fixed with $4 \%$ paraformaldehyde for $15 \mathrm{~min}$ at room temperature and stained with $0.2 \%$ crystal violet for $20 \mathrm{~min}$ (Solarbio, Beijing) at room temperature. The number of colonies visible to the naked eye (each colony contained $>50$ cells; $1 \times$ magnification) was counted using ImageJ v2 software (Image); National Institutes of Health).

\section{CCK-8 assay}

Cell proliferation was also analysed with the Cell Counting Kit (CCK)-8 assay (Solarbio, Beijing). A375 cells were collected, washed, resuspended, counted using a haemocytometer, added to 96 -well plates at 2000 cells/well and cultured at $37{ }^{\circ} \mathrm{C}$ and $5 \% \mathrm{CO}_{2}$. Cell proliferation was analysed every day for 4 days. Briefly, $10 \mu \mathrm{g}$ CCK-8 solution ( $5 \mathrm{mg} / \mathrm{ml}$ ) was added to the cells $(10 \mu \mathrm{l} /$ well $)$, followed by incubation for $2 \mathrm{~h}$. Finally, absorbance at $450 \mathrm{~nm}$ was measured with a microplate reader (DeTie, Nanjing, China).

\section{Cell apoptosis analysis using flow cytometry}

Apoptotic cells were detected using an Annexin V-FITC Apoptosis Detection Kit (eBioscience, San Diego, CA, USA) according to the manufacturer's instructions. Cells were harvested and washed with PBS. Cell suspensions were prepared at a final density of $1 \times 10^{6} / \mathrm{ml}$, and a 100 $\mu \mathrm{l}$ aliquot was mixed with $5 \mu \mathrm{l}$ annexin V-FITC and $5 \mu \mathrm{l}$
PI staining solution, followed by incubation at room temperature for 10-15 min. Apoptotic cells were detected by flow cytometry (Beckman Coulter, Inc.), and the apoptosis data were analysed using FlowJo v10 software (FlowJo LLC).

\section{Immunohistochemistry}

Tissue samples were fixed in $4 \%$ paraformaldehyde, embedded in paraffin, and sectioned at a thickness of $5 \mu \mathrm{m}$. Immunohistochemistry staining was analysed by Wuhan Servicebio Technology Co., Ltd. The sections were examined and photographed under a microscope (magnification, 200×; Olympus Corporation) in five random fields of view per sample, and an experienced pathologist test the sections. The expression level of HSDL2 was divided into 4 groups based on the staining intensity ( 0 , negative; 1 , low; 2 , medium; and 3 , high). In addition, the ratio of stained cells was as follows: $0,0 \%$ stained cells; $1,1-25 \%$ stained cells; $2,26-50 \%$ stained cells; and 3,51-100\% stained cells. A staining index (score, $0-12$ ) was examined by the staining intensity $\times$ the score for the positive area.

\section{Tumour growth in vivo assay}

Female BALB/c nu/nu athymic nude mice (7-9 weeks of age) were purchased from Beijing Vital River Laboratory Animal Technology Co., Ltd. The housing environment was kept between 16 and $26{ }^{\circ} \mathrm{C}$ with relative humidity between 30 and $70 \%$ under a regular 12-h light, 12-h dark cycle. All experimental procedures were approved by the Institutional Animal Care and Use Committee (IACUC) of the First Affiliated Hospital of Wannan Medical College.

For the tumour growth assay, 12 female BALB/c nu/ nu athymic nude mice were randomly divided into two groups ( $n=6 /$ group), and then $1 \times 10^{7}$ A375 cells stably transfected with control or HSDL2 shRNA lentivirus were subcutaneously injected into the right flanks of female nude mice. After 4 weeks, the experiments were finished, and the mice were sacrificed and dissected at the endpoint. Then, the volume and weight of the tumours were measured.

For $\mathrm{CuE}$ treatment in vivo, $1 \times 10^{7} \mathrm{~A} 375$ cells were subcutaneously injected into the right flanks of female nude mice. When the tumour size reached approximately $0.5 \mathrm{~cm}$ in diameter, the mice were assigned into two groups ( $n=6 /$ group) by a random numbered table: (1) Control group injected intraperitoneally with $100 \mu \mathrm{l}$ PBS; (2) CuE group (intraperitoneally administered with $\mathrm{CuE}, 5 \mathrm{mg} / \mathrm{kg}$ ) one times per four days. After 3 weeks, the mice were sacrificed and dissected at the endpoint, and then, the volume and weight of the tumours were measured. In addition, the nude mice were divided into four 
group ( $\mathrm{n}=6$ /group): (1) Control group; (2) $\mathrm{CuE}$ group; (3) HSDL2 group; (4) HSDL2 + CuE group. $1 \times 10^{7} \mathrm{~A} 375$ cells stably transfected with control or HSDL2-overexpressing lentivirus were subcutaneously injected into the right flanks of female nude mice, and then the tumourbearing mice were intraperitoneally injected with $\mathrm{CuE}$ $(5 \mathrm{mg} / \mathrm{kg}$ ) one times per four days. After 4 weeks, the mice were sacrificed and dissected at the endpoint, and then, the volume and weight of the tumors were measured.

\section{Statistical analysis}

All experiments were performed with at least three replicates. SPSS v21.0 software (SPSS Inc., Chicago, IL, USA) was used for statistical analysis. Data are presented as the mean \pm standard deviation of three independent experiments. One-way analysis of variance or unpaired two-tailed Student's $\mathrm{t}$ test was used for data. $\mathrm{P}<0.05$ was considered statistically significant.

\section{Result}

\section{HSDL2 expression in melanoma and adjacent normal} tissues

HSDL2 expression was examined in melanoma and adjacent normal tissues by immunohistochemistry. HSDL2 was upregulated in melanoma (Fig. 1A), which was confirmed by the higher rate of immunopositivity relative to nontumour tissue (Fig. 1B). Importantly, Kaplan-Meier survival curve analysis demonstrated that there was a marked association between higher expression of HSDL2 and worse melanoma patient survival (Fig. 1C).

\section{Function of HSDL2 in melanoma}

To investigate HSDL2 function in melanoma, A375 cells were infected with an HSDL2-overexpressing lentivirus or shRNA targeting HSDL2 lentivirus. The expression of HSDL2 in A375 cells after infection is shown in Fig. 2. HSDL2 lentivirus infection elevated HSDL2 expression (Fig. 2A, B), while shRNA-HSDL2 infection inhibited HSDL2 expression (Fig. 2C, D). The above results demonstrated that HSDL2 could be successfully overexpressed or knocked down in A375 cells.

Next, CCK-8 assays and colony formation assays were used to evaluate cell proliferation. Upregulation of HSDL2 promoted A375 cell proliferation, while downregulation of HSDL2 inhibited cell proliferation (Fig. 3A, B). Subsequently, apoptosis assays were also used to test whether HSDL2 could affect apoptosis in addition to inhibiting proliferation. The apoptotic fraction was decreased in HSDL2-overexpressing cells relative to control cells, while the apoptotic fraction was increased in HSDL2-depleted cells relative to control cells (Fig. 3C). Additionally, the relative apoptotic
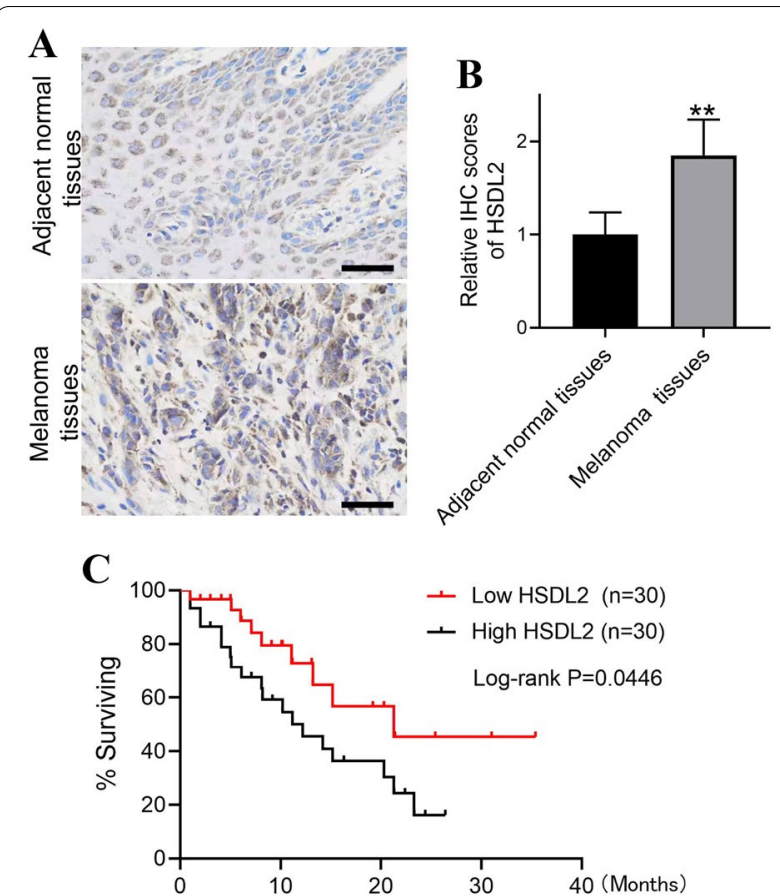

Fig. 1 HSDL2 protein expression in melanoma and adjacent normal tissue samples detected by immunohistochemistry. A Representative image of HSDL2 IHC staining in the 60 paired melanoma tissue and adjacent normal tissues (scale bar $=200 \mu \mathrm{m}$ ). B The expression of HSDL2 in melanoma tissue and adjacent normal tissues based on the results of IHC was quantified. C Kaplan-Meier survival curve of HSDL2 in melanoma according to the expression of HSDL2. The results are presented by the mean $\pm S E M$; ${ }^{* * P}<0.01$, compared with the control group

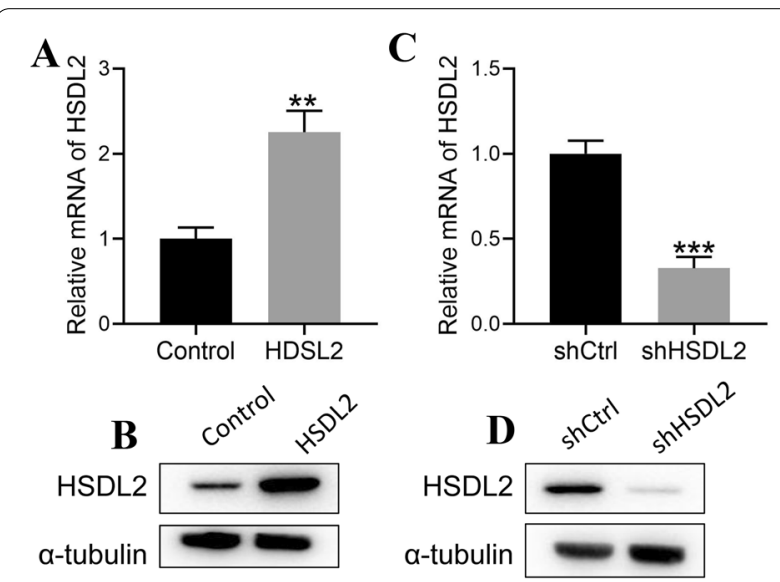

Fig. 2 Efficiency of HSDL2 expression in A375 cells using HSDL2-overexpressing lentivirus or shRNA-targeting HSDL2 lentivirus. A, B The expression of HSDL2 was examined in HSDL2-overexpressing A375 cells via RT-qPCR and western blotting. C, D The expression of HSDL2 was detected in HSDL2-silenced A375 cells via RT-qPCR and western blotting. The experiments were repeated three times. The results are presented as the mean $\pm S E M$; ${ }^{* *} P<0.01$ and ${ }^{* * *} \mathrm{P}<0.001$, compared with the control group 
A
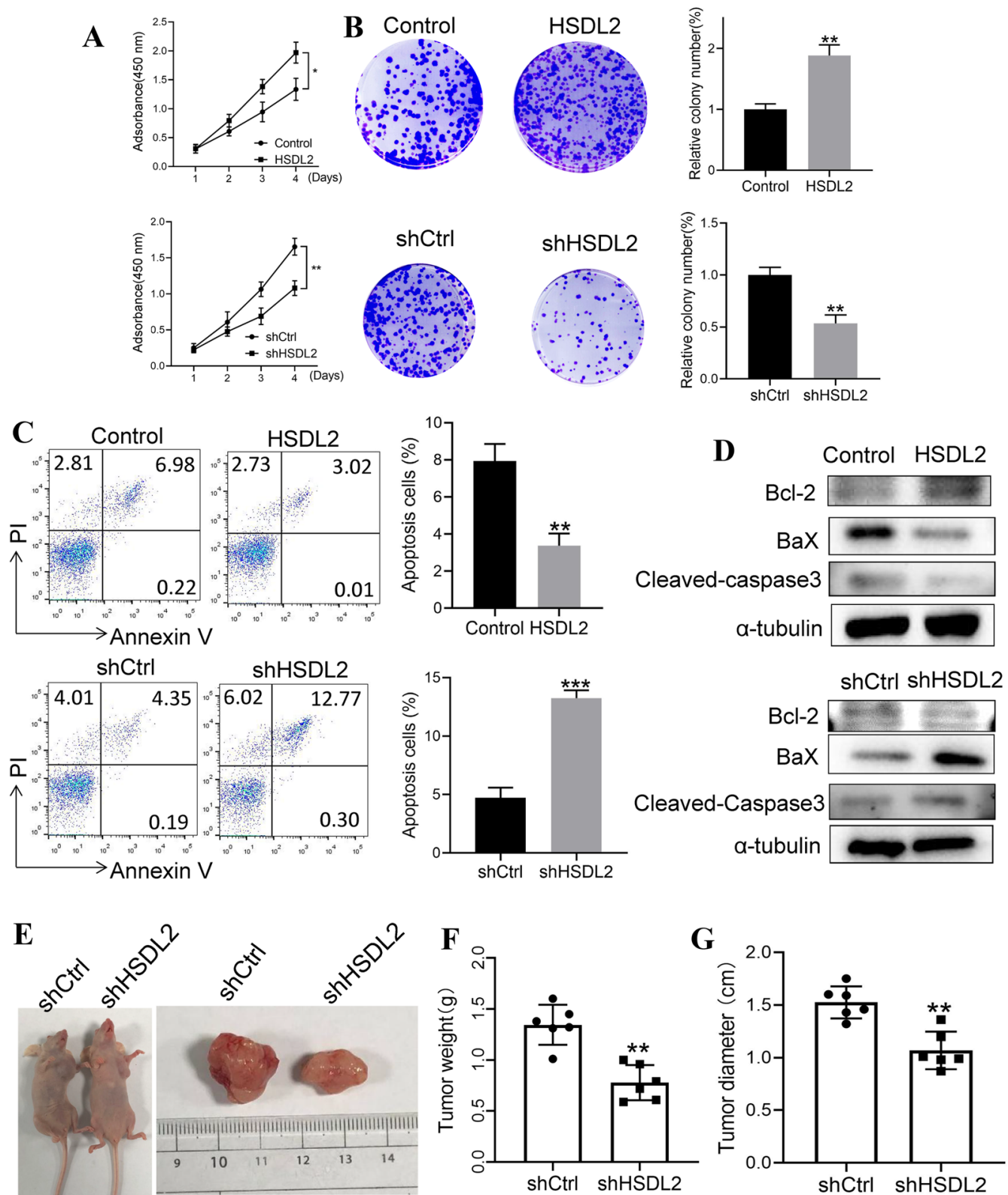

Fig. $3 \mathrm{HSDL} 2$ promotes the growth of melanoma in vitro and in vivo. HSDL2 was overexpressed or silenced in A375 cells, and A CCK-8 assays were performed. B Colony forming assays were performed. Imaging and quantification of colony formation assays were performed between HSDL2-silenced A375 cells or HSDL2-overexpressing A375 cells and control cells. C Apoptotic cells were tested by FACS analysis using an Annexin V-FITC Apoptosis Detection Kit. D Apoptosis markers ( $\mathrm{BaX}, \mathrm{BCl}-2$ and cleaved-Caspase3) were detected by western blotting. E-G A375 cells with silenced HSDL2 were subcutaneously injected into nude mice. After 4 weeks, the mice were sacrificed and dissected at the endpoint. Tumour growth and weight were examined $\left(n=6 /\right.$ group). The experiments were repeated three times. All data represent the mean $\pm S E M,{ }^{*} \mathrm{P}<0.05$, ${ }^{* *} \mathrm{P}<0.01$ and ${ }^{* * *} \mathrm{P}<0.001$, compared with the control group

proteins were also investigated, and the results shown in Fig. 3D, overexpression of HSDL2 in A375 cells significantly enhanced the expression of $\mathrm{Bcl} 2$, markedly inhibited the expression of $\mathrm{BaX}$ and reduced the levels of cleaved caspase-3 relative to control cells, while the expression of $\mathrm{Bcl} 2$ was also downregulated, and the levels of $\mathrm{BaX}$ and cleaved caspase- 3 were increased in HSDL2-depleted cells.

Finally, a subcutaneous tumour xenografting assay in $\mathrm{BALB} / \mathrm{c}$ nude mice demonstrated that stably silencing HSDL2 in A375 cells significantly suppressed tumour growth. Much smaller tumours were formed 
by HSDL2-depleted A375 cells, and the average tumour weight and volume at the end of the experiment were markedly decreased in HSDL2-depleted cells relative to control cells (Fig. 3E-G). Taken together, these in vitro and in vivo results suggested that HSDL2 promotes melanoma cell proliferation and inhibits melanoma cell apoptosis.

\section{HSDL2 knockdown inhibited the AKT and ERK signaling pathways in melanoma cells}

To investigate the mechanisms by which HSDL2 regulated melanoma growth, we examined the activation of multiple signal transduction pathways involved in cell growth and survival in A375 cells after HSDL2 knockdown using the PathScan Stress and Apoptosis Signaling Antibody Array Kit and RTK Signaling Antibody Array Kit. HSDL2 silencing markedly reduced the phosphorylation of ERK1/2 (Thr202/Tyr204) and AKT (Ser473) relative to cells infected with shCtrl lentivirus (Fig. 4A), suggesting that cell growth inhibition in the absence of HSDL2 is due to inactivation of ERK and Akt signalling.
The phosphorylation levels of ERK1/2 and AKT were also verified in HSDL2-overexpressing or HSDL2depleted cells. As shown in Fig. 4B, C, the upregulation of HSDL2 significantly enhanced the phosphorylation levels of ERK1/2 and AKT relative to control cells, while the downregulation of HSDL2 markedly suppressed the phosphorylation levels of ERK1/2 and AKT relative to the control cells. These results showed that HSDL2 promotes proliferation and inhibits apoptosis in melanoma by activating the ERK1/2 and AKT pathways.

\section{HSDL2 is a target of cucurbitacin E}

Since HSDL2 had a positive effect on melanoma growth, we attempted to identify a regulator of HSDL2 expression. Hence, we screened some compounds from a natural compound library to assess whether they could regulate the expression of HSDL2. As depicted in Fig. 5A, $\mathrm{B}$ and Additional file 1: Fig. S1A, B, the results from RTqPCR and western blotting assays demonstrated that $\mathrm{CuE}$ could inhibit the mRNA and protein expression of HSDL2.

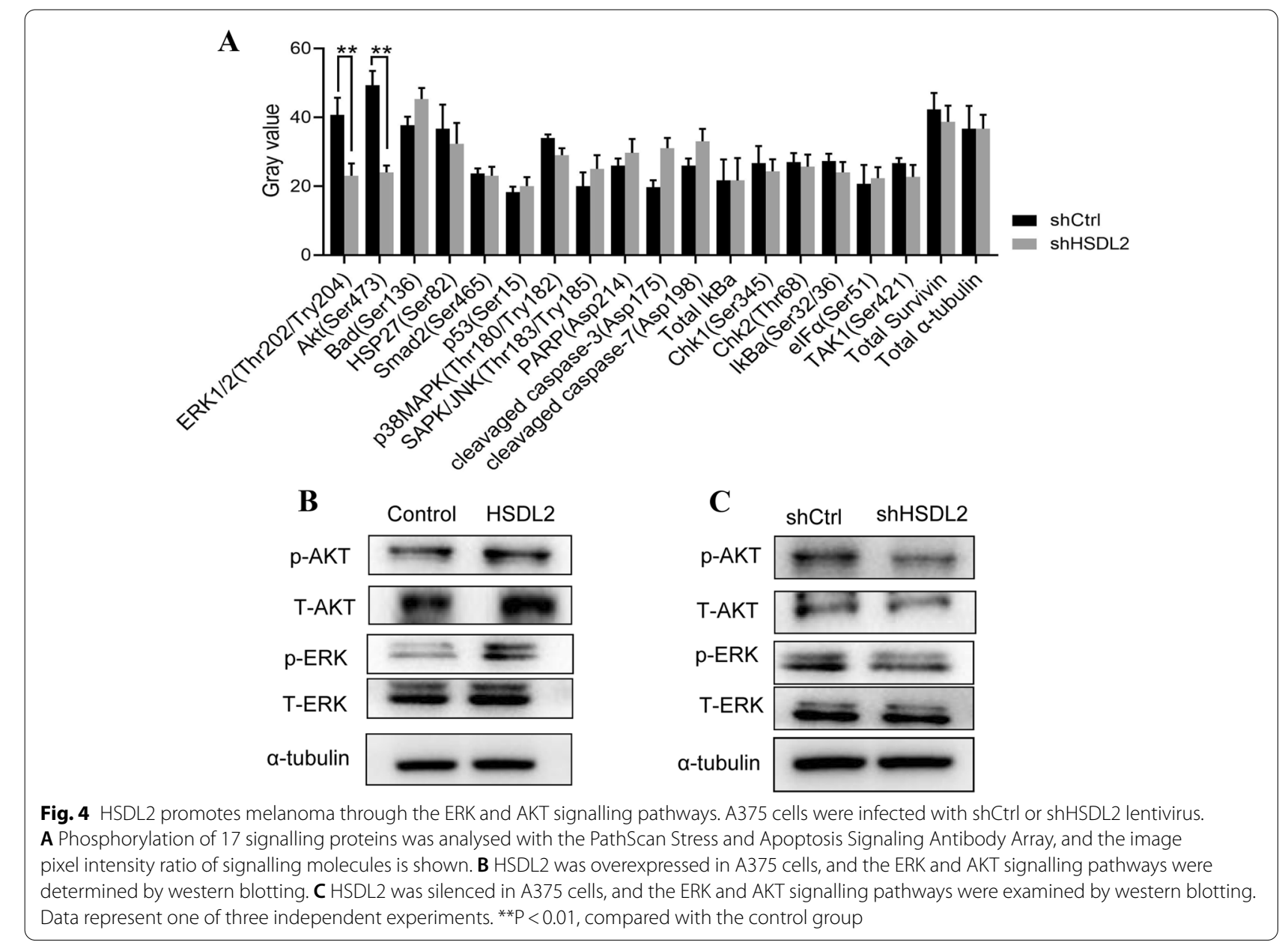




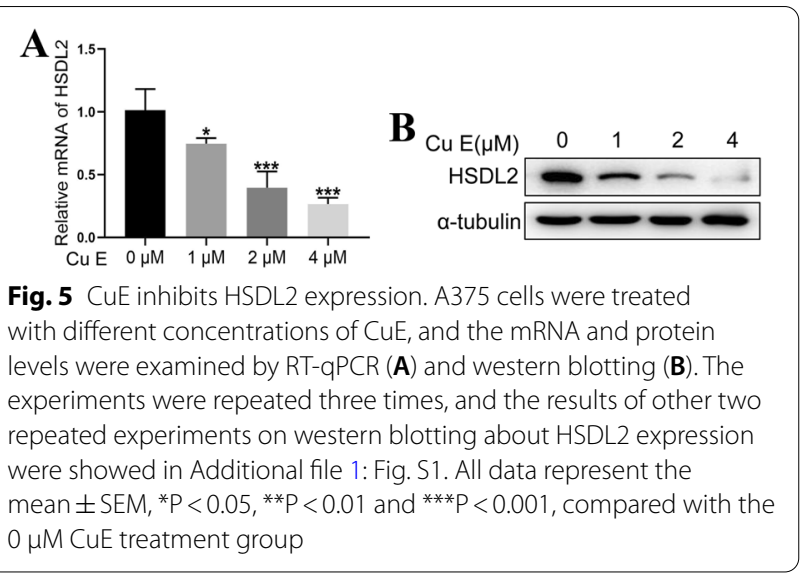

\section{Cucurbitacin $\mathrm{E}$ impedes proliferation and induces apoptosis in melanoma cells}

Cell proliferation and apoptosis assays were performed to confirm the anticancer effects of cucurbitacin E. A CCK- 8 assay was performed with A375 cells exposed to different doses of $\mathrm{CuE}(0,1,2,4$ and $8 \mu \mathrm{M})$. As shown in Fig. $6 \mathrm{~A}, \mathrm{~B}, \mathrm{CuE}$ inhibited A375 cell proliferation in a dose-and time-dependent manner, and the marked death of A375 cells was induced by $4 \mu \mathrm{M} \mathrm{CuE}$. Therefore, $\mathrm{CuE}$ at a dose of $2 \mu \mathrm{M}$ was used for subsequent experiments. Colony formation assays showed that $\mathrm{CuE}$ remarkably reduced the number of colonies formed by A375 cells (Fig. 6C, D). Next, the effect of CuE on apoptosis in A375 cells was tested by flow cytometry and relative apoptotic
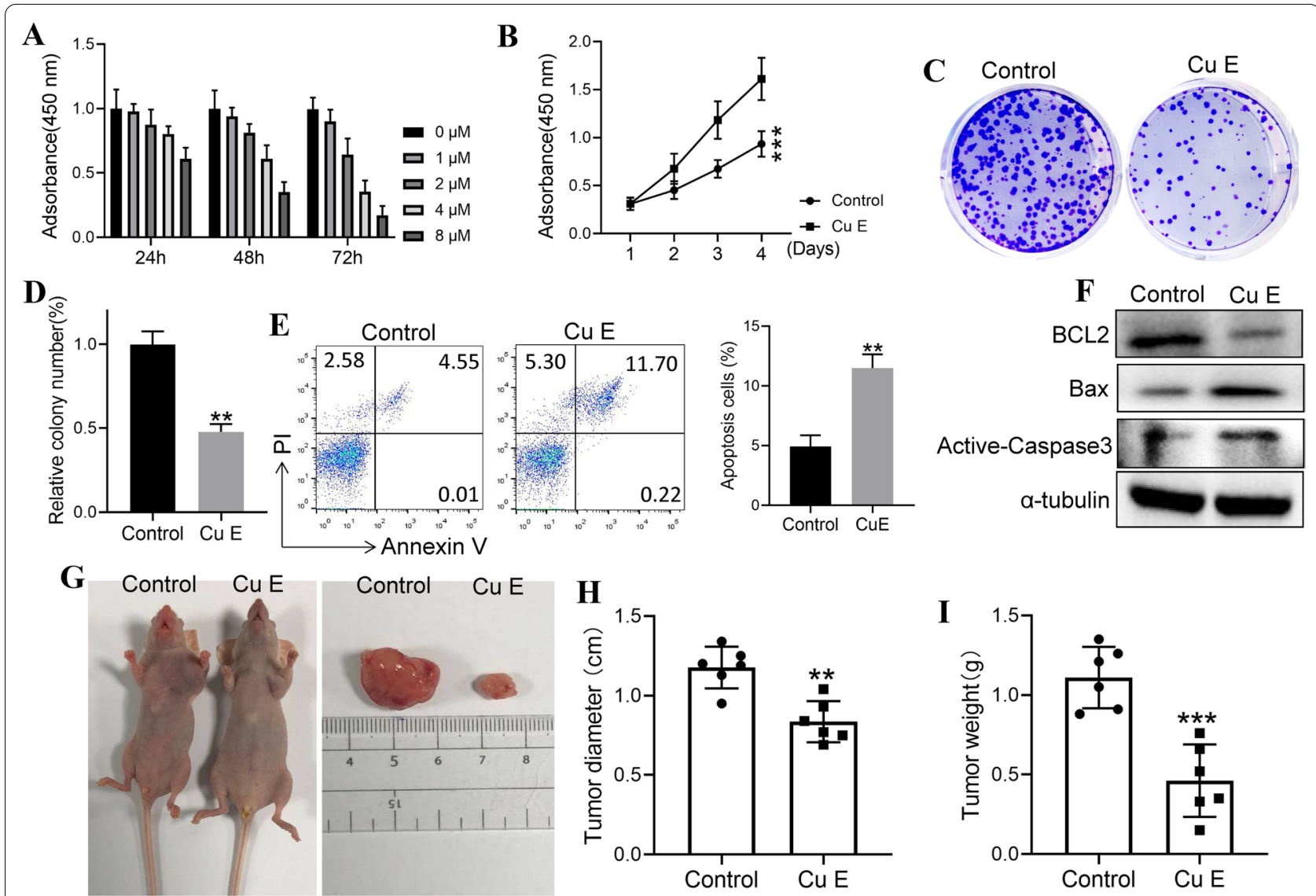

Fig. 6 CuE inhibits the growth of melanoma in vitro and in vivo. The inhibitory function of CuE in the proliferation of A375 cells was examined in vitro (A), and then $4.0 \mu \mathrm{M}$ CUE was used to analyse the proliferation rate by CCK-8 assay (B). $2.0 \mu \mathrm{M}$ CuE was used to analyse the colony formation ability by colony forming assay (C, D). A375 cells were treated with CuE $(2.0 \mu \mathrm{M})$, and $24 \mathrm{~h}$ after treatment, cells were analysed by FACS for cell apoptosis (E). Immunoblotting analysis of Bcl2, BaX and cleaved caspase-3 in A375 cells treated with CuE (F). A375 cells were subcutaneously injected into nude mice. When the tumour size reached approximately $0.5 \mathrm{~cm}$ in diameter, the tumour-bearing mice were intraperitoneally injected with CuE $(5 \mathrm{mg} / \mathrm{kg}$ ). For 3 weeks, the mice were sacrificed and dissected at the endpoint. Tumour growth and weight were examined ( $n=6 / \mathrm{group}$, G-I). The experiments were repeated three times. All data represent the mean $\pm S E M ;{ }^{* *} \mathrm{P}<0.01$ and ${ }^{* * *} \mathrm{P}<0.001$ compared with the control group 
protein assays. The results showed that after treatment with $\mathrm{CuE}$, the number of apoptotic cells had significantly increased (Fig. 6E). Similarly, the expression of Bcl2 was also downregulated, and the levels of $\mathrm{BaX}$ and cleaved caspase- 3 were upregulated in the cells treated with $\mathrm{CuE}$ at $48 \mathrm{~h}$ (Fig. 6F). In addition, the antitumour activity of $\mathrm{CuE}$ in vivo was also assessed using xenografts in BALB/c nude mice, as shown in Fig. 6G-I. CuE significantly slowed tumour growth, and the average weight and volume of tumours at the end of the experiment in the $\mathrm{CuE}$ group were less than those in the control group. Taken together, these data suggested that $\mathrm{CuE}$ treatment significantly inhibits melanoma growth by suppressing melanoma cell proliferation and enhancing melanoma cell apoptosis.

\section{Cucurbitacin E suppresses proliferation and induces apoptosis in melanoma cells by downregulating HSDL2} Our results showed that $\mathrm{CuE}$ effectively suppressed the growth of melanoma cells and inhibited the expression of HSDL2. However, whether $\mathrm{CuE}$ displays its antitumour activity through mediating the level of HSDL2 remained unknown. Thus, we treated HSDL2-overexpressing A375 cells with $\mathrm{CuE}$. RT-qPCR and western blot assays showed higher HSDL2 expression in HSDL2-overexpressing cells treated with $\mathrm{CuE}$ than in cells treated with $\mathrm{CuE}$ alone (Fig. 7A). The data concluded from CCK-8 assays that HSDL2-overexpressing cells had heightened cell abilities compared to cells treated with $\mathrm{CuE}$ alone after culture with $\mathrm{CuE}$ for $48 \mathrm{~h}$ (Fig. 7B). Subsequently, a colony formation assay demonstrated that the inhibitory effect of $\mathrm{CuE}$ on clone formation was also weakened after HSDL2 upregulation (Fig. 7C). In addition, the number of apoptotic cells was also decreased in HSDL2-overexpressing cells treated with $\mathrm{CuE}$ (Fig. 7D). Similarly, the expression of $\mathrm{Bcl} 2$ was also upregulated, and the levels of $\mathrm{BaX}$ and cleaved caspase-3 were suppressed in the HSDL2-overexpressing cells treated with $\mathrm{CuE}$ (Fig. 7E). Moreover, in the subcutaneous tumour xenografting assay, upregulation of HSDL2 promoted tumour growth, and the overexpression of HSDL2 slowed the antitumour activity of $\mathrm{CuE}$ (Fig. 7F-H). There were no changes in the average weight or volume of tumours between HSDL2-overexpressing cells treated with $\mathrm{CuE}$ and cells treated with $\mathrm{CuE}$ alone (Fig. 7F-H).

\section{CuE inhibits the AKT and ERK signalling pathways in melanoma cells}

Our previous data indicated that $\mathrm{CuE}$ could inhibit the expression of HSDL2; hence, we investigated whether $\mathrm{CuE}$ also affected the AKT and ERK signalling pathways. We performed western blotting assays to investigate the change in the phosphorylation levels of AKT and ERK.
The data showed that $\mathrm{CuE}$ markedly reduced the phosphorylation levels of ERK1/2 and AKT relative to control cells (Fig. 8A). Moreover, upregulation of HSDL2 enhanced ERK1/2 and AKT phosphorylation induced by $\mathrm{CuE}$ (Fig. 8B). Thus, the above data suggested that $\mathrm{CuE}$ inhibits melanoma cells by blocking the HSDL2-mediated AKT and ERK pathways.

\section{Discussion}

A previous study showed that HSDL2 expression was upregulated in gliomas and was positively correlated with tumour grade [22]. This suggests that HSDL2 is involved not only in tumorigenesis but also in tumour progression. However, little is known about the expression and function(s) of HSDL2 in melanoma. Our data show that HSDL2 is overexpressed in melanoma relative to adjacent normal tissue. In vitro and in vivo experiments revealed that HSDL2 is important for melanoma growth. Combined with HSDL2 knockdown and overexpression assays in melanoma cells, the data confirmed the positive role of HSDL2 in melanoma proliferation and apoptotic inhibition, suggesting that silencing HSDL2 may be a potential strategy for blocking melanoma progression.

To investigate the molecular basis for the effects of HSDL2 on melanoma cell proliferation and survival, we examined the activation status of multiple intracellular signalling proteins involved in cell survival and proliferation using stress, apoptosis, and RTK signalling arrays. HSDL2 knockdown decreased the phosphorylation of ERK1/2 (Thr202/Tyr204) and AKT (Ser473) relative to control cells. The ERK and Akt signalling pathways are constitutively activated in melanoma and play an important role in melanoma development and progression [23, 24]. Activated ERK1/2 can regulate the relative proteins involved in proliferation and apoptosis, such as Bad, Bcl2 , and C-myc, thereby contributing to melanoma growth $[25,26]$. In addition, the Akt pathway is another significant pathway in melanomagenesis, promoting melanoma cell proliferation, metastasis and drug resistance, inhibiting apoptosis, and stimulating DNA mutation [27, 28] and is associated with poor survival [29]. Therefore, our results provide the first evidence of a causal relationship between HSDL2 expression and the ERK and Akt signalling pathways in human melanoma development.

Some natural compounds that could inhibit the expression of HSDL2 to slow melanoma progression would provide new prospects and methods for the treatment of patients with melanomas. Previous reports have also shown that $\mathrm{CuE}$ has remarkable potential in suppressing the growth of multiple cancer cell types [18, 30,31]. CuE can inhibit cell proliferation, induce cell cycle arrest and enhance apoptotic cell death by modulating the STAT3, MAPK, PI3K/AKT, Wnt/beta-catenin and mTOR 


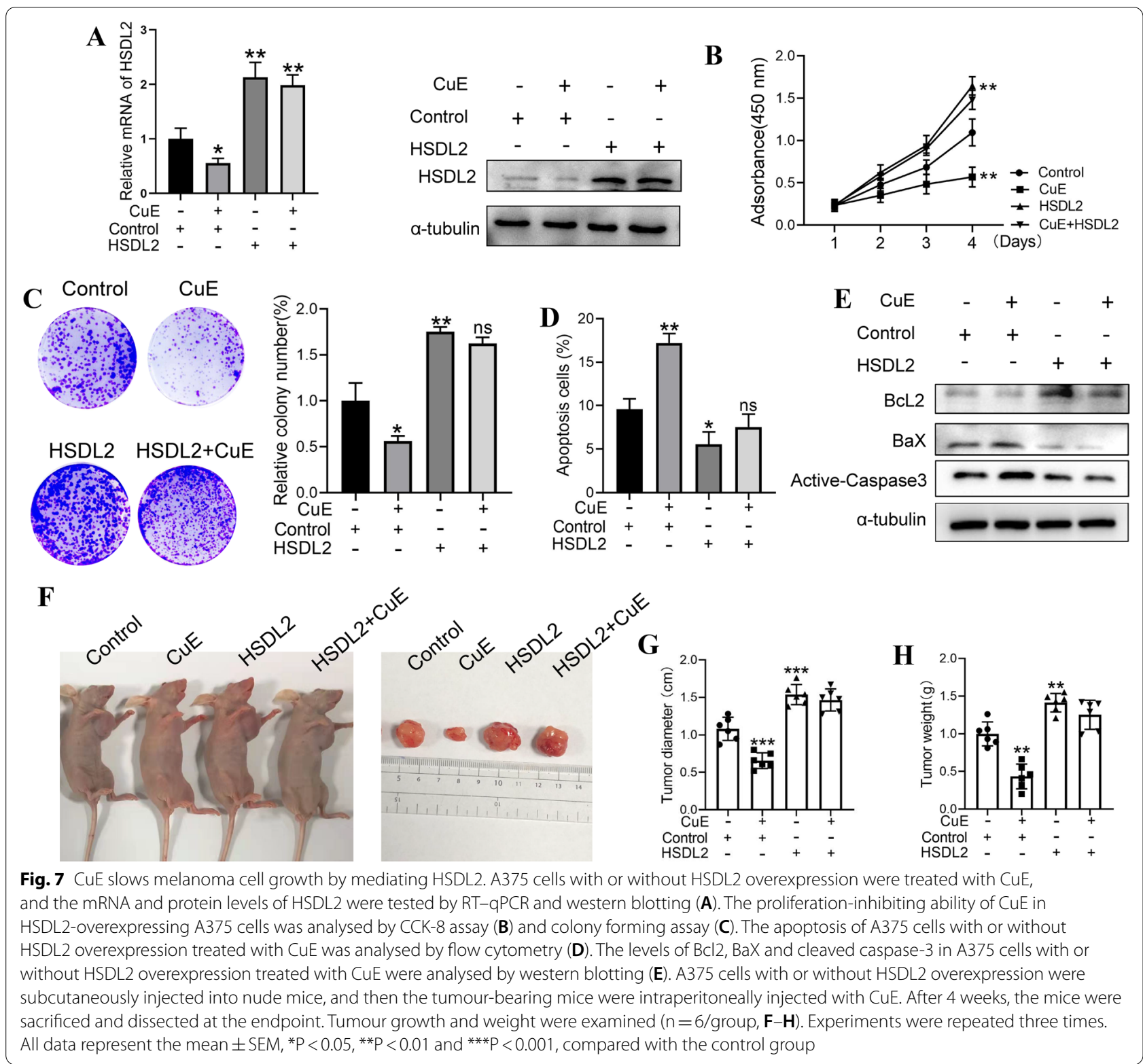

signalling pathways $[17,19,32-34]$. CuE can also suppress angiogenesis by inhibiting the VEGFR2-mediated JAK2 - STAT3 signalling pathway and cancer cell metastasis by inhibiting depolymerization of actin filaments [35, 36]. In addition, $\mathrm{CuE}$ can enhance the sensitivity of tumours such as gastric cancer and colorectal cancer to conventional chemotherapeutic drugs $[17,18]$. Fortunately, our data demonstrated that $\mathrm{CuE}$ could inhibit HSDL2 expression and melanoma cell proliferation and induce cell apoptosis, a significant target for slowing tumour development, by suppressing the ERK and AKT pathways and reducing xenograft melanoma growth. Moreover, upregulation of HSDL2 slowed the antitumour activity of $\mathrm{CuE}$, and weakened $\mathrm{CuE}$-inhibited the phosphorylation of ERK and AKT. In sum, these results support the potential development of $\mathrm{CuE}$ as a therapeutic drug for melanoma.

\section{Conclusion}

Our data demonstrated that HSDL2 is overexpressed in melanoma tissues and that its silencing blocks melanoma progression by inhibiting cell proliferation and enhancing apoptosis via inhibition of the ERK and AKT pathways. Therefore, HSDL2 may be a promising therapeutic target against melanoma. Moreover, $\mathrm{CuE}$ inhibited melanoma growth by blocking the 


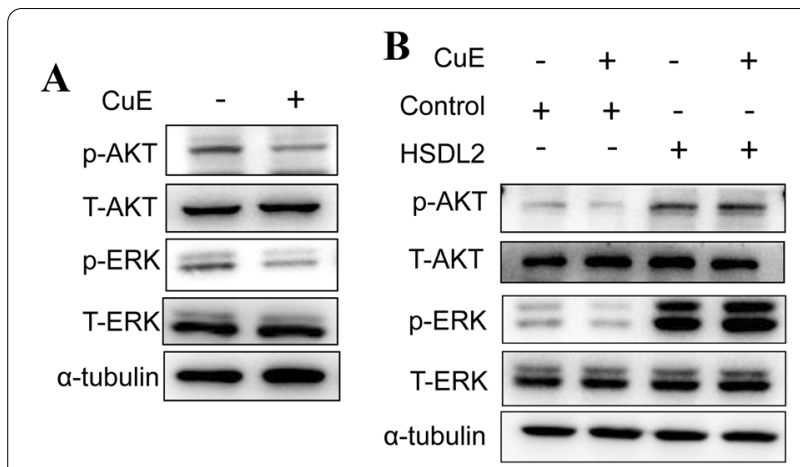

Fig. 8 CuE inhibits the ERK and AKT signalling pathways by downregulating HSDL2. A375 cells were treated with CUE for $24 \mathrm{~h}$, and the phosphorylation levels of ERK and AKT were determined by western blotting (A). A375 cells with or without HSDL2 overexpression were treated with $\mathrm{CuE}$, and the phosphorylation levels of ERK and AKT were determined by western blotting (B). Data represent one of three independent experiments and KJ2020A0599), Grants for Scientific Research of BSKY from Anhui Medical University (No. XJ201903), the Foundation of the Key Laboratory of Colleges and Universities in Anhui Province (No. RNA202008) and the Research Fund of Anhui Medical University (no. 2019xkj006).

\section{Availability of data and materials}

The datasets used and/or analysed during the current study are available from the corresponding author on reasonable request.

\section{Declarations}

Ethics approval and consent to participate

Informed consent was obtained from all participants, and the experimental protocol was approved by the Ethics Committee of the First Affiliated Hospital of Wannan Medical College.

\section{Consent for publication}

Not applicable.

\section{Competing interests}

The authors report no conflicts of interest in relation to this work.

Received: 2 December 2021 Accepted: 3 February 2022

Published online: 22 February 2022

HSDL2-mediated ERK and AKT pathways. Our results suggest that $\mathrm{CuE}$ may be a useful chemopreventive strategy for melanoma patients with high HSDL2 levels.

\begin{abstract}
Abbreviations
BCl-2: B-cell lymphoma/leukaemia-2; Bax: Bcl-2 associated X protein; CuE: Cucurbitacin E; ERK: Extracellular signal-regulated kinase; FBS: Foetal bovine serum; SDR: Short-chain dehydrogenase/reductase; HSDL2: Hydroxysteroid dehydrogenase-like 2; JAK: Janus kinase; MAPK: Mitogen-activated protein kinase; mTOR: Mammalian target of rapamycin; PBS: Phosphate-buffered saline; PI3K: Phosphoinositide 3-kinase; RT-qPCR: Real-time reverse transcription-quantitative polymerase chain reaction:; STAT3: Signal transducer and activator of transcription 3; VEGFR2: Vascular endothelial growth factor receptor 2 .
\end{abstract}

\section{Supplementary Information}

The online version contains supplementary material available at https://doi. org/10.1186/s13020-022-00582-y.

Additional file 1: Fig. S1. CuE suppresses HSDL2 protein level. (A and B) A375 cells were treated with different doses of $\mathrm{CuE}$, and the protein levels were examined by western blotting. (A) the results of the second repeat experiment; (B) the results of the third repeat experiment.

\section{Acknowledgements}

\section{Not applicable.}

\section{Authors' contributions}

$J M$, and DL designed the experiments. WL, HW and LC conducted experiments on the clinical samples and provided material support. WL, LC and BT performed most of the experiments. WL and GK drafted the manuscript. SW and YS contributed to the analysis and interpretation of the data. WL and HW modified the manuscript. All authors read and approved the final manuscript.

\section{Funding}

This work was supported by grants from the National Natural Science Foundation of Anhui Province, China (No. 2008085QH377), the Colleges and Universities in Anhui Province Natural Science Research Projects (KJ2019A0418

\section{References}

1. Sun FD, Wang PC, Shang J, Zou SH, Du X. Ibrutinib presents antitumor activity in skin cancer and induces autophagy. Eur Rev Med Pharmacol Sci. 2018;22(2):561-6.

2. Carr S, Smith C, Wernberg J. Epidemiology and risk factors of melanoma. Surg Clin N Am. 2020;100(1):1-12.

3. Li C, Zhang Q, Li Z, Feng S, Luo H, Liu R, Wang L, Geng Y, Zhao X, Yang Z, et al. Efficacy and safety of carbon-ion radiotherapy for the malignant melanoma: a systematic review. Cancer Med. 2020;9(15):5293-305.

4. Zhou H, Wu J, Leng S, Hou C, Mo L, Xie X, Wang L, Xu Y. Knockdown of circular RNA VANGL1 inhibits TGF-beta-induced epithelial-mesenchymal transition in melanoma cells by sponging miR-150-5p. J Cell Mol Med. 2021;25:10827.

5. Miller AJ, Mihm MC Jr. Melanoma. N Engl J Med. 2006;355(1):51-65.

6. Berrocal A, Cabanas L, Espinosa E, Fernandez-de-Misa R, Martin-Algarra S, Martinez-Cedres JC, Rios-Buceta L, Rodriguez-Peralto JL. Melanoma: diagnosis, staging, and treatment, Consensus group recommendations. Adv Ther. 2014;31(9):945-60.

7. Yang $Y$, Han A, Wang X, Yin X, Cui M, Lin Z. Lipid metabolism regulator human hydroxysteroid dehydrogenase-like 2 (HSDL2) modulates cervical cancer cell proliferation and metastasis. J Cell Mol Med.

8. Cheng Z, Li Y, Sui C, Sun X, Xie Y. Synthesis, purification and crystallographic studies of the C-terminal sterol carrier protein type 2 (SCP-2) domain of human hydroxysteroid dehydrogenase-like protein 2. Acta crystallogr Sect F Struct Biol Commun. 2015;71(Pt 7):901-5.

9. Gronemeyer T, Wiese S, Ofman R, Bunse C, Pawlas M, Hayen H, Eisenacher M, Stephan C, Meyer HE, Waterham HR, et al. The proteome of human liver peroxisomes: identification of five new peroxisomal constituents by a label-free quantitative proteomics survey. PLoS ONE. 2013;8(2):e57395.

10 Bian X, Liu R, Meng Y, Xing D, Xu D, Lu Z. Lipid metabolism and cancer. J Exp Med. 2021;218:1.

11. Ding $X$, Zhang $W$, Li S, Yang $H$. The role of cholesterol metabolism in cancer. Am J Cancer Res. 2019;9(2):219-27.

12. Han A, Xu R, Liu Y, Yin X, Lin Z, Yang W. HSDL2 acts as a promoter in pancreatic cancer by regulating cell proliferation and lipid metabolism. Onco Targets Ther. 2021;14:435-44.

13. Dong B, Yang Y, Han A, Zhang S, Lin Z, Wang Y, Piao J. Ectopic expression of HSDL2 is related to cell proliferation and prognosis in breast cancer. Cancer Manag Res. 2019;11:6531-42.

14. Zeng J, Ma X, Wang J, Liu R, Shao Y, Hou Y, Li Z, Fang Y. Down-regulated HSDL2 expression suppresses cell proliferation and promotes apoptosis in papillary thyroid carcinoma. Biosci Rep. 2019;39:6. 2021;25(10):4846-59. 
15. Jia X, Huang C, Hu Y, Wu Q, Liu F, Nie W, Chen H, Li X, Dong Z, Liu K. Cirsiliol targets tyrosine kinase 2 to inhibit esophageal squamous cell carcinoma growth in vitro and in vivo. J Exp Clin Cancer Res. 2021;40(1):105.

16. Arel-Dubeau AM, Longpre F, Bournival J, Tremblay C, Demers-Lamarche J, Haskova P, Attard E, Germain M, Martinoli MG. Cucurbitacin E has neuroprotective properties and autophagic modulating activities on dopaminergic neurons. Oxidat Med Cell Longevity. 2014;2014:425496.

17 Yang P, Liu W, Fu R, Ding GB, Amin S, Li Z. Cucurbitacin E Chemosensitizes Colorectal Cancer Cells via Mitigating TFAP4/Wnt/beta-Catenin Signaling. J Agric Food Chem. 2020;9:78.

18. Si W, Lyu J, Liu Z, Wang C, Huang J, Jiang L, Ma T. Cucurbitacin E inhibits cellular proliferation and enhances the chemo-response in gastric cancer by suppressing AKt activation. J Cancer. 2019;10(23):5843-51.

19. Jing SY, Wu ZD, Zhang TH, Zhang J, Wei ZY. In vitro antitumor effect of cucurbitacin E on human lung cancer cell line and its molecular mechanism. Chin J Nat Med. 2020;18(7):483-90.

20. Kong Y, Chen J, Zhou Z, Xia H, Qiu MH, Chen C. Cucurbitacin E induces cell cycle G2/M phase arrest and apoptosis in triple negative breast cancer. PLoS ONE. 2014;9(7):e103760.

21. Shan $X L$, Zhou $X Y$, Yang J, Wang $Y L$, Deng $Y H$, Zhang $M X$. Inhibitory effect of cucurbitacin $\mathrm{E}$ on the proliferation of ovarian cancer cells and its mechanism. Chin J Cancer. 2010;29(1):20-4.

22. Ruokun C, Yake X, Fengdong Y, Xinting W, Laijun S, Xianzhi L. Lentivirusmediated silencing of HSDL2 suppresses cell proliferation in human gliomas. Tumour Biol. 2016;37(11):15065-77.

23. Tang $R, X u X$, Yang W, Yu W, Hou S, Xuan Y, Tang Z, Zhao S, Chen Y, Xiao X, et al. MED27 promotes melanoma growth by targeting AKT/MAPK and NF-kappaB/iNOS signaling pathways. Cancer Lett. 2016;373(1):77-87.

24. Pearlman RL. Montes de Oca MK, Pal HC, Afaq F: Potential therapeutic targets of epithelial-mesenchymal transition in melanoma. Cancer Lett. 2017:391:125-40.

25. Tsai WB, Aiba I, Long Y, Lin HK, Feun L, Savaraj N, Kuo MT. Activation of Ras/PI3K/ERK pathway induces c-Myc stabilization to upregulate argininosuccinate synthetase, leading to arginine deiminase resistance in melanoma cells. Cancer Res. 2012;72(10):2622-33.

26. Lee CW, Yen FL, Ko HH, Li SY, Chiang YC, Lee MH, Tsai MH, Hsu LF. Cudraflavone C induces apoptosis of A375.S2 melanoma cells through mitochondrial ROS production and MAPK activation. Int J Mol Sci. 2017:18:7.

27. Castro MV, Barbero GA, Villanueva MB, Grumolato L, Nsengimana J, Newton-Bishop J, Illescas E, Quezada MJ, Lopez-Bergami P. ROR2 has a protective role in melanoma by inhibiting Akt activity, cell-cycle progression, and proliferation. J Biomed Sci. 2021;28(1):76

28. Ma J, Wang H, Guo S, Yi X, Zhao T, Liu Y, Shi Q, Gao T, Li C, Guo W. A20 promotes melanoma progression via the activation of Akt pathway. Cell Death Dis. 2020;11(9):794.

29. Robertson GP. Functional and therapeutic significance of Akt deregulation in malignant melanoma. Cancer Metastasis Rev. 2005;24(2):273-85.

30. Song H, Wang Y, Li L, Sui H, Wang P, Wang F. Cucurbitacin E inhibits proliferation and migration of intestinal epithelial cells via activating cofilin. Front Physiol. 2018;9:1090.

31. Zhang $L$, Liang $H$, Xin Y. Cucurbitacin E inhibits esophageal carcinoma cell proliferation, migration, and invasion by suppressing Rac1 expression through PI3K/AKT/mTOR pathway. Anticancer Drugs. 2020;31(8):847-55.

32. Liu Y, Yang H, Guo Q, Liu T, Jiang Y, Zhao M, Zeng K, Tu P. Cucurbitacin E Inhibits Huh7 Hepatoma Carcinoma Cell Proliferation and Metastasis via Suppressing MAPKs and JAK/STAT3 Pathways. Molecules. 2020;25:3.

33. Wang $Y, X u S$, Wu Y, Zhang J. Cucurbitacin E inhibits osteosarcoma cells proliferation and invasion through attenuation of PI3K/AKT/mTOR signalling pathway. Biosci Rep. 2016;36:6.

34. He X, Gao Q, Qiang Y, Guo W, Ma Y. Cucurbitacin E induces apoptosis of human prostate cancer cells via cofilin-1 and mTORC1. Oncol Lett. 2017;13(6):4905-10

35. Dong Y, Lu B, Zhang X, Zhang J, Lai L, Li D, Wu Y, Song Y, Luo J, Pang X, et al. Cucurbitacin $E$, a tetracyclic triterpenes compound from Chinese medicine, inhibits tumor angiogenesis through VEGFR2-mediated Jak2STAT3 signaling pathway. Carcinogenesis. 2010:31(12):2097-104.

36. Sorensen PM, lacob RE, Fritzsche M, Engen JR, Brieher WM, Charras G, Eggert US. The natural product cucurbitacin E inhibits depolymerization of actin filaments. ACS Chem Biol. 2012;7(9):1502-8.

\section{Publisher's Note}

Springer Nature remains neutral with regard to jurisdictional claims in published maps and institutional affiliations.
Ready to submit your research? Choose BMC and benefit from:

- fast, convenient online submission

- thorough peer review by experienced researchers in your field

- rapid publication on acceptance

- support for research data, including large and complex data types

- gold Open Access which fosters wider collaboration and increased citations

- maximum visibility for your research: over $100 \mathrm{M}$ website views per year

At BMC, research is always in progress.

Learn more biomedcentral.com/submissions 